\title{
Psychotherapieforschung: Die Spitze des Eisbergs und der Rest
}

Peter Schulthess

Anlässlich des Psy-Kongresses in Bern (vgl. Tagungsbericht in diesem Heft) hat Jens Gaab einen bemerkenswerten Vortrag zur Psychotherapieforschung gehalten. Jens Gaab ist Professor für Klinische Psychologie und Psychotherapie an der Universität Basel. Im Folgenden werden seine Ausführungen zusammengefasst wiedergegeben.

In der Psychotherapieforschung ist seit deren Beginn eine Unmenge an Daten zusammengetragen worden. Lagen 1909 gerade mal zwei Studien vor, so entstehen heute gemäss pubmed.gov (einer Art Google der medizinisch-klinischen Forschung) 8.000 bis 9.000 pro Jahr, ein äusserst aktives Forscherverhalten also. Ein Motor dieses Forschungsaktivismus ist die Annahme, dass es für psychische Störungen spezifische Ursachen und spezifische Behandlungen gibt. Diese Annahme geht auf Freud zurück, der so die Psychoanalyse (Psychotherapie) in das System der Medizin einfügen wollte.

$\mathrm{Da}$ alle Therapierichtungen $\mathrm{zu}$ ihrer wissenschaftlichen Anerkennung bis heute belegen müssen, dass sie gleichwertige Wirksamkeit mit anderen Verfahren haben (oder gar noch wirksamer sind, noch spezifischer wirken), beflügelt das die Forschung ungemein als einen Wettbewerb, wer noch besser und noch wirksamer sei.

Interessanterweise können erste Annahmen zur Psychotherapie auf Franz Anton Mesmers Annahme eines animalischen Magnetismus zurückgeführt werden. Heute kennen wir etwa 200 verschiedene Psychotherapieverfahren und 500 Methoden. Die Society of Clinical Psychology (eine Division der APA) ordnet die Verfahren und Methoden nach den Gesichtspunkten der evidenzbasierten Medizin und hat Kriterien entwickelt, die für die Evidenzbasierung gelten.

An der Hypothese, dass psychische Störungen spezifische Ursachen haben und mit spezifischen Behandlungen geheilt werden könnten, kamen forschungsbasiert erste Zweifel auf, indem angenommen wurde, dass unterschiedliche Therapieverfahren in etwa gleiche Erfolge zeigen (Dodo-Bird-Verdikt). Diese von Rosenzweig 1936 publizierte Annahme war die Grundlage für die Formulierung von allgemeinen beziehungsweise gemeinsamen Wirkfaktoren der unterschiedlichen Therapiekonzepte. Wie war das zu erklären, dass Therapiekonzepte mit unterschiedlichen, gar sich widersprechenden Theorien $\mathrm{zu}$ gleichwertigen Behandlungserfolgen führten? Es war ein erster Hinweis darauf, dass es wohl in der Psychotherapie andere Wirkfaktoren geben müsse als spezifische Interventionen für spezifische Störungen. Es wurde gefolgert, dass die Methoden egal bzw. austauschbar seien und dass es der Glaube des Therapeuten an die Wirksamkeit seiner spezifischen Interventionen ist, der den Erfolg ermöglicht.

Der Referent benutzte das Bild eines Eisberges, um zu zeigen, dass es nicht reicht, wenn Forschung bloss die aus dem Wasser ragende Spitze untersucht und die 91,68 Prozent des Volumens, das unter dem Wasser liegt, vernachlässigt. Was aber ist, auf Interventionen bezogen, das, was unter der Wasseroberfläche liegt? Das Placebo! Placebo wurde möglicherweise zuerst von der katholischen Kirche zur Diagnose von Besessenheit durch den Teufel eingesetzt. Man spritzte Weihwasser auf eine zu begutachtende Person. Wenn sie dann schrie und tobte, war das der Beleg für die Besessenheit und Anwesenheit des Teufels, was die Indikation für einen Teufelsaustreibungsprozess bedeutete. Es wurde dann vergleichsweise auch gewöhnliches Wasser ver-
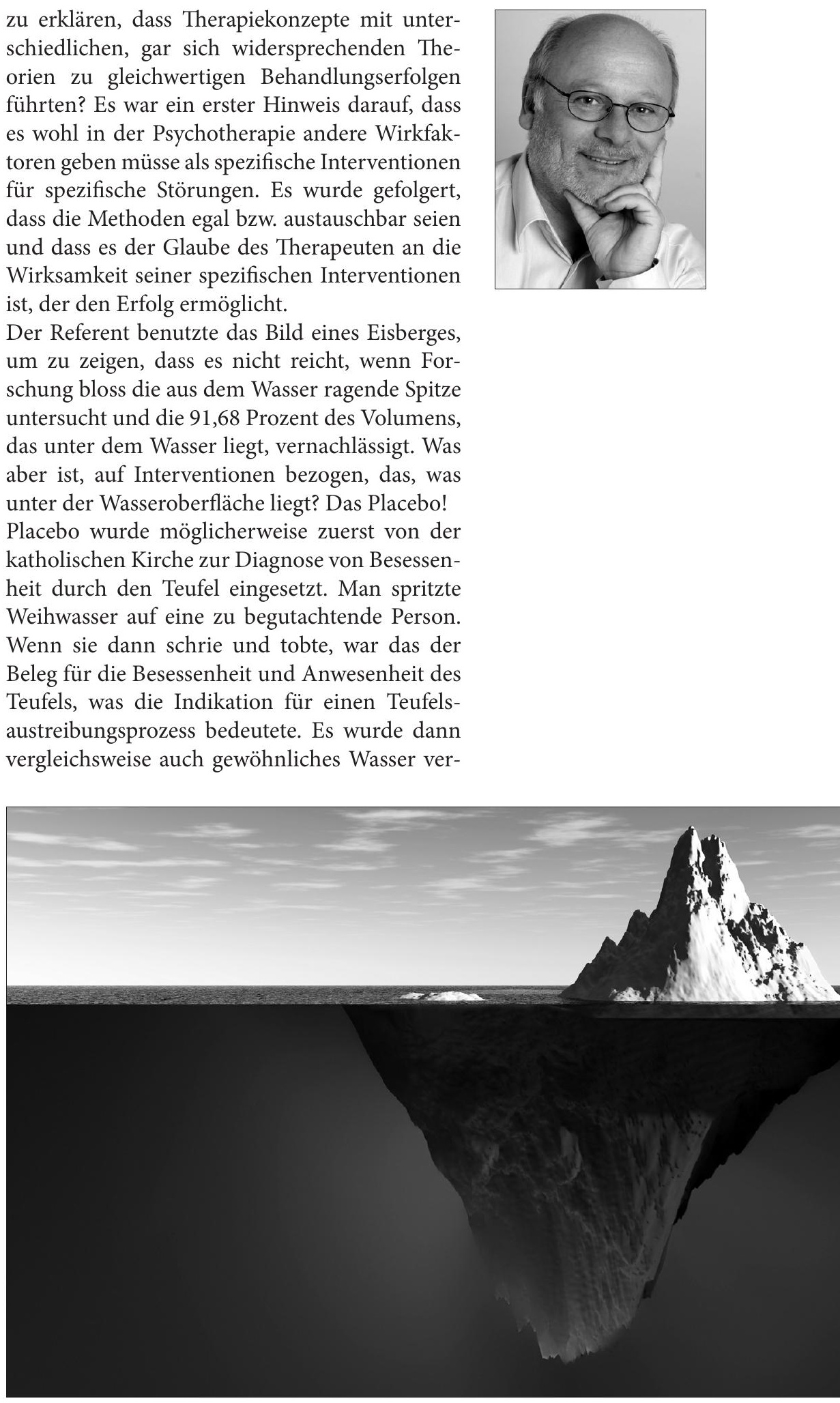
spritzt. Wenn die Person ebenfalls schrie und tobte, dann war das ein Beleg, dass nicht der Teufel in ihr sei, sondern dass sie verrückt sei. Sie wurde verwahrt. So hat Placebo manches Leben gerettet (Kaptchuk et al., 2009).

In Versuchen mit Mesmers Magnetismus unter Louis XVI. wurde gezeigt, dass die gewünschte Heil-Wirkung auch dann eintrat, wenn gar keine Person hinter dem Vorhang war, sondern die Versuchsperson das bloss glaubte, da ihr das so gesagt wurde (Franklin et al., 2008 [1784]).

Placebo-Studien bei Operationen (man führte die Operation gar nicht durch, sondern schnitt bloss oberflächlich und nähte wieder zu) zeigten, dass Placebo in 65 Prozent der Fälle wirksam war. In der Behandlung von Schmerzpatienten gar in 80 Prozent. Die Forschung zeigt einen zunehmenden Trend der Wirksamkeitserhöhung des Placebos, je nachdem wie es verabreicht wird (Jonas et al., 2015). Versuche mit Antidepressiva zeigen, dass das Placebo in 75 Prozent der Fälle wirksam ist (Khan \& Brown, 2015).

Es wurden auch Versuche im nicht-medizinischen Bereich gemacht: Professionellen Geigern wurden Stradivari Geigen und gewöhnliche marktgängige Geigen zum Spielen mitgegeben. Wenn der Geiger nicht wusste, dass er auf einer Stradivari spielte, beurteilte er das Instrument als schlechter als die handelsüblichen (Fritz et al., 2012). Versuche mit Wein zeigten, dass Versuchspersonen denselben Wein als besser einschätzten, wenn man ihnen sagte, er sei sehr teuer, als wenn man ihnen sagte, es handle ich um einen billigen Wein (Plassmann et al., 2008). Auch mit Putzfrauen wurde ein Placebo-Experiment gemacht. Sie wurden in zwei Gruppen aufgeteilt. Die eine hatte einfach ihre Putz-Aufgabe zu verrichten. Der anderen sagte man, Putzen sei Sport und sehr gesund. Beide Gruppen taten dieselbe Arbeit. Es zeigten sich verblüffende Unterschiede: Die «Sportlerinnen» nahmen an Gewicht ab, ihr BMI wurde reduziert, der Blutdruck und der Fettanteil im Gewebe sanken.

In einer anderen Studie wurde gezeigt, dass die regelmässige Einnahme eines Placebos über längere Zeit die Mortalität senkte. Das Risiko war um die Hälfte geringer als bei denen, die das Placebo nicht regelmässig einnahmen (Crum \& Langer, 2007).

Die Erwartung scheint also die Wirkung zu beeinflussen. Doch nicht nur das: Es sind auch die
Beziehungen zum Therapeuten (Versuchsleiter) und dem «Wirkstoff», die die Wirkung beeinflussen. In der Folge wurde die Fragestellung verändert: Es ist offenbar das «Bedeutung-Geben», das ein Wirkfaktor ist, denn Placebo an sich hat keine Wirkung (Moerman \& Jonas, 2002).

Was das mit Psychotherapie zu tun hat? Mit Medikamenten kann man vergleichsweise einfach kontrollierte Placebo-Studien durchführen. In der Psychotherapie ist das schwieriger. In kontrollierten Studien, wo eine Gruppe mit spezifischer Psychotherapie behandelt wurde und eine Kontrollgruppe lediglich eine non-direktive, supportive und empathische "Therapie» erhielt, zeigte sich, dass bei struktureller Äquivalenz der Vergleichsgruppen hinsichtlich Anzahl, Format und Dauer der Therapiesitzungen, sowie der Ausbildung des Therapeuten und inhaltlichen Restriktionen, gleichwertige Resultate erreicht wurden. Unterschiede ergaben sich nur, wenn die beiden Gruppen strukturell nicht äquivalent waren (Baskin et al., 2003).

Eine weitere Studie kommt zu dem Schluss, dass bei der Wirksamkeit von Psychotherapie lediglich 1 Prozent spezifischen Faktoren zuzuschreiben ist, 34 Prozent Faktoren ausserhalb der Therapie und 65 Prozent unspezifischen Faktoren (Cuijpers et al., 2012).

Da muss man schon sehr überzeugt sein, um weiterhin an der These festzuhalten, dass psychische Störungen eine spezifische Ursache hätten und mit einem spezifischen Therapievorgehen behandelt werden könnten. Auch neuere Studien belegen, dass das Dodo-Verdikt weiterhin gilt (Leichsenring et al., 2014).

Ist Psychotherapie Placebo? Der Referent zog ein Zwischenfazit:

- spezifische Effekte von Psychotherapie sind dann möglich, wenn man die Kontrollbedingungen entsprechend gestaltet

- bei fairen Vergleichen ist der Unterschied nicht bedeutsam

- Psychotherapien unterscheiden sich nur theoretisch, nicht aber in ihrer Wirkung

- Placebos mit psychotherapeutischer Wirkung sind möglich und wirksam (mit mittleren bis grossen Effekten)

- Überrascht?

Im Folgenden kam Gaab auf Rosenzweig zurück, der als Erster über implizite gemeinsame Faktoren der verschiedenen Therapiekonzepte 
publizierte und führte dann weiter zu Jerome Frank (1986), welcher die Psychotherapie als Bedeutungstransformation beschrieb. Er bezog sich auf die "grosse Psychotherapiedebatte» und verwies auf die eben erschienene deutsche Ausgabe des Buches Die Psychotherapie Debatte. Was Psychotherapie wirksam macht (Wampold et al., 2018). Darin wird ein Überblick über die Psychotherapieforschung gegeben und das Kontextmodell von Wampold dargestellt, welches zum Fazit kommt, dass übergreifende Faktoren, wie etwa die Therapeuteneigenschaften, für die Wirksamkeit der Psychotherapie eine wichtige Rolle spielen. Die Autoren zeigen auf, dass Psychotherapie wirkt. Klinisch bedeutsame Wirkfaktoren sind: gemeinsame Ziele von Patient und Therapeut, Empathie, gute Beziehung, Wertschätzung. Im Mittelfeld rangieren: Persönlichkeit des Therapeuten, Echtheit, Adaption, Erwartung. Vernachlässigbar sind: Unterschiede der Therapierichtungen, Kompetenz, Methoden, Adhärenz (Wampold \& Imel, 2015). Das sind Wirkfaktoren, die schon Carl Rogers beschrieb (Rogers, 2007 [1957]).

In einer eigenen, noch unveröffentlichten Studie zur Placeboforschung in der Psychotherapie konnte er zeigen, dass es keine Rolle spielt, ob das Placebo verdeckt oder offen als Placebo verabreicht wird. Bei offener Abgabe und entsprechenden Erläuterungen des Therapeuten entfaltete sich gar eine grössere Wirkung als bei verdeckter. Dies ist ein Hinweis darauf, dass die Beziehung ein «Verum» ist (Gaab et al., 2018).

Aus theoretischer Perspektive steht Psychotherapie in einer unangenehmen Nähe zu Placeboeffekten. Wie sich daraus befreien, um sich als seriöse Wissenschaft etablieren zu können? Es reicht nach Meinung des Referenten nicht, sich auf empirische Evidenz, klinische Expertise und Erwartungen der Patienten abzustützen, vielmehr bräuchte es zusätzlich ethische Prinzipien, die sich ausrichten auf: Autonomie, Benefizienz, Non-Malefizienz und Gerechtigkeit. Er verwies hier auf eine neue Publikation zur Psychotherapieethik (Trachsel et al., 2018).

$\mathrm{Zu}$ den ethischen Prinzipien gehört, die Patienten offen aufzuklären über das, was wir als Therapeuten wissen und was wir tun, aber auch darüber, was wir nicht wissen. Entscheidend für den Therapierfolg ist eine offene, vertrauenswürdige und partnerschaftliche Beziehung.
In der Diskussion wurde gefragt, ob sich die Forschung nicht einfach noch mehr anstrengen müsse, um die spezifischen Wirksamkeitsfaktoren besser zu erfassen. Gaab antwortete mit der Frage, warum man denn weiterhin so sehr auf die Spitze des Eisberges schauen wolle? Die künftige Psychotherapieforschung solle sich doch besser auf die 92,68 Prozent des Eisberges konzentrieren, die unterhalb des Sichtbaren liegen.

\section{Literatur}

Baskin, T.W., Tierney, S.C., Minami, T. \& Wampold, B. E. (2003). Establishing specificity in psychotherapy: a meta-analysis of structural equivalence of placebo controls. Journal of Consulting and Clinical Psychology, 71(6), 973-979.

Crum, A. J. \& Langer, E. J. (2007). Mind-set matters. Exercise and the placebo effect. Psychological Science, 18(2), 165-171.

Cuijpers, P., Driessen, E., Hollon, S. D., van Oppen, P., Barth, J. \& Andersson, G. (2012). The efficacy of non-directive supportive therapy for adult depression: a meta-analysis. Clinical psychology review, 32(4), 280-291.

Frank, J.D. (1986). Psychotherapy - the transformation of meanings: discussion paper. Journal of the Royal Society of Medicine, 79(6), 341-346.

Franklin, B., Majault, M. J., Le Roy, J.B., Sallin, C.L., Bailly J.-S., d’Arcet, J., de Bory, G., Guillotin J.-I. \& Lavoisier, A. (2008 [1784]). Report of the commissioners charged by the king with the examination of animal magnetism. International Journal of Clinical and Experimental Hypnosis, 50(4), 332-363.

Fritz, C., Curtin, J., Poitevineau, J., Morrel-Samuels, P. $\&$ Tao, F. C. (2012). Players preferences among new and old violins. Proceedings of the National Academy of Sciences, 109(3), 760-763.

Gaab, J., Locher, C. \& Blease, C. (2018). Placebo and Psychotherapy: Differences, Similarities and Implications. International review of neurobiology, 138, 241-255.

Jonas, W. B., Crawford, C., Colloca, L., Kapchuk, T.J., Moseley, B., Miller F. G., Kriston, L., Linde, K. \& Meissner, K. (2015). To what extent are surgery and invasive procedures effective beyond a placebo response? A systematic review with meta-analysis of randomized, sham controlled trials. $B M J$ open, 5(12), e009655. https://doi.org/10.1136/bmjopen-2015-009655 
Khan, A. \& Brown, W.A. (2015). Antidepressants versus placebo in major depression: an overview. World Psychiatry, 14(3), 294-300.

Kaptchuk, T. J., Kerr C. E. \& Zanger, A. (2009). Placebo controls, exorcism and the devil. The Lancet, 374(9697), 1234-1245.

Leichsenring, F., Salzer, S., Beutel, M.E., Herpertz, S., Hiller, W., Hoyer, J. \& Huesing, J. (2014). Long-term outcome of psychodynamic therapy and cognitivebehavioral therapy in social anxiety disorder. American Journal of Psychiatry, 171(10), 1074-1082.

Moerman, D. E. \& Jonas, W. B. (2002). Deconstructing the placebo effect and finding the meaning response. Annals of Internal medicine, 136(6), 471-476.

Plassmann, H., O’Doherty, J., Shiv, B. \& Rangel, A. (2008). Marketing actions can modulate neural representations of experienced pleasantness. Proceedings of the National Academy of Sciences, 105(3), 1050-1054.
Rogers, C. R. (2007 [1957|). The necessary and sufficient conditions of therapeutic personality change. Psychotherapy: Theory, Research, Practice, Training, 44(3), 240-248.

Rosenzweig, S. (1936). Some implicit common factors in diverse methods of psychotherapy. American Journal of Orthopsychiatry, 6(3), 412-415.

Trachsel, M., Gaab, J. \& Biller-Andorno, N. (2018). Ethische Standards in der Psychotherapie. Göttingen: Hogrefe.

Wampold, B. E. \& Imel, Z.E. (2015). The great psychotherapy debate: The evidence for what makes psychotherapy work. New York: Routledge.

Wampold, B. E., Imel, Z. E. \& Flückiger, C. (2018). Die Psychotherapiedebatte. Was Psychotherapie wirksam macht. Göttingen: Hogrefe.

Peter Schulthess ist Vorstandsmitglied der ASP.

\section{Computergestützte Psychotherapie}

\section{Chancen und Risiken}

Peter Schulthess

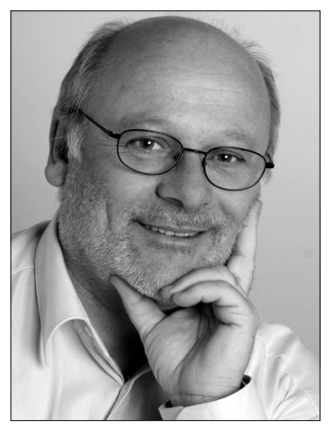

Wie im Bericht über den Psy-Kongress 2018 erwähnt, ist das Referat von Dirk Helbling zum Thema «Einsatz von Cognitive Computing in der Psychotherapie: Chancen und Risiken» eine ausführliche Zusammenfassung wert. Dirk Helbling ist Professor of Computational Social Science an der ETH Zürich.

Das Thema muss uns Psychotherapeuten echt beschäftigen und zu denken geben, wir können es nicht einfach ignorieren, da Cognitive Computing, so wie in anderen Bereichen des Gesundheitswesens, auch in der Psychotherapie zur Anwendung kommen wird bzw. bereits in Anwendung ist.

IT-basierte Datenmengen ermöglichen neue Möglichkeiten zur Optimierung der Leistungsfähigkeit der Medizin, sie bergen aber auch Risiken. Stichworte sind: Personalisierte Medizin, optimierte Diagnosestellung und Behandlungsassistenz anhand von Leitlinien. Und natürlich haben an der Nutzung dieser Daten auch die Kostenträger ihr Interesse zur Senkung der Ge- sundheitskosten oder zur Risikoselektion der Versicherer.

Das World Wide Web (WWW) ist ein weltweites Nachrichtennetz (ursprünglich für militärische nachrichtendienstliche Zwecke erfunden) und bildet einen globalen Markt für Daten. Soziale Netzwerke sind Knoten im WWW, wo Daten generiert und weiter geliefert werden. Die Datenmenge ist derart immens, dass einzelne Personen diese unmöglich auswerten können. Zur Auswertung wird künstliche Intelligenz eingesetzt. In einer Minute erfolgen weltweit 700.000 Google-Abfragen und 500.000 Facebook-Posts. Unsere Bewegungen erzeugen Datenspuren, die sogar in Echtzeit eingesehen werden können.

Das Big Data Paradigma lautete: Wenn wir nur genügend Daten hätten, dann bräuchten wir keine Theorien mehr, die Daten würden die Wahrheit enthüllen. Seither werden so viele Daten wie möglich gesammelt, um Gehirn und Psyche zu verstehen. Das ist ein Traum und zugleich ein Albtraum! 